\title{
EDITORIAL
}

\section{Injury prevention and safety promotion}

$\mathrm{D}$ uring March 15th-18th, Mexico will be the centre of the injury and violence prevention universe. Not only does it welcome over 1500 scientific experts from all over the world to the $9^{\text {th }}$ World Conference on Injury Prevention and Safety Promotion, but it also welcomes the Ministers of Health of the Americas for their first summit on injuries and violence prevention and about 80 Ministry of Health Focal Points for Violence and Injury Prevention to their second global meeting.

This special issue of Salud Pública de México complements the very rich scientific programme of the $9^{\text {th }}$ World Conference on Injury Prevention and Safety Promotion. I welcome this initiative, which has allowed young researchers from around the world to collaborate with several leading experts on innovative papers. Having this special issue published in Spanish and English helps overcome one of the barriers often faced by those less familiar with the English language who want to share their scientific work with others.

This special issue shows a clear evolution in the field of injuries and violence prevention. Most papers have been written by authors from low- and middle-income countries. The papers cover a wide range of issues -including descriptive epidemiology, studies of risk factors such as alcohol, injury prevention, trauma care, and larger policy issues and strategies. As time passes and national and international policy makers increasingly recognize the importance of injury prevention, additional experts are being empowered all over the world to conduct research on programmes and publish results.

World Conferences always represent a good opportunity to reflect on the status of the field. To the findings and ideas presented in this issue, I would like to add some reflections based on discussions that took place in recent meetings with WHO colleagues and close partners in WHO Collaborating Centres as well as through regular contact with colleagues in many countries.

The field of injuries and violence prevention -although still in its infancy, as pointed out in this is- sue-is gaining momentum and has grown considerably in the past decades. International policy discussions on injuries and violence prevention and treatment have taken place more than ten times in the last decade at the highest political levels, namely within the UN General Assembly and the World Health Assembly. Dozens of countries have developed national strategies and/or plans of action. More than 100 Ministries of Health have appointed focal persons for injuries and violence prevention in the last five years and some have started creating units within the ministry with their own resources.

Although far from sufficient, our knowledge about the magnitude and preventability of the problem has also increased considerably. The Global Burden of Disease in the 1990's clearly showed the magnitude of the problem in relation to other causes of ill health. Studies such as the Adverse Childhood Experiences (ACE) study, the WHO Multi-Country Study on Violence against Women and many others, have given us considerably more insight on the far reaching health consequences of injuries and violence. In addition more and more countries are able to provide at least some information on the economic costs of injuries. Evidence on the effectiveness of prevention programmes has been growing steadily, primarily in high income, but increasingly in low- and middle-income countries.

Financial resources -although still too little- have grown steadily. Governments, through their ministries of health, transport, justice or international cooperation, as well as foundations and even private companies, have started injecting resources into the field.

WHO has tried to support the growth of the field by providing technical assistance, supporting the exchange of information and capacity building, developing world reports and guidance documents and conducting advocacy. Over the coming years it will continue to invest in such activities while undertaking particular efforts in five strategic areas. 
Developing model country programmes: One of the most compelling arguments to bring to the attention of policy makers is the documentation of success and cost-effectiveness of programmes in neighbouring countries or provinces. Coordinated and focused efforts are needed to develop and show the success of a range of comprehensive violence and injury prevention programmes in low- and middle-income countries. At present, technical support to low- and middle-income countries is often focussed on small scale programmes with limited resources. This allows for the development of a small data collection system or some prevention effort, but often does not result in a programme that has been shown to save lives. Efforts are needed to develop programmes that show substantial savings in human and economic terms. This requires well planned programmes in select tipping-point countries conducted in close collaboration among national governments, donor agencies, and international groups able to provide technical assistance. Advocacy, communications and marketing: Despite successful events such as the launches of major new reports or studies, World Health Days or Road Safety Weeks, the field still lacks considerable advocacy strength. A large part of the progress made in fields such as HIV prevention or tobacco control is due to the sustained efforts at local, national and international levels -often championed by NGOs representing those affected and their families or professionals in the field. Statistics and data on the magnitude and cost effectiveness of interventions are very important, but these need to be complemented by the "human face" of injury and violence. Strong and organized NGOs of victims and survivors of burns, child abuse, youth and intimate partner violence, road traffic crashes or other types of injuries are needed in all countries. In addition, an active and visible international NGO representing the professionals working in this field is also needed. It is hoped that the International Society for Violence and Injury Prevention (ISVIP) will continue to grow into playing that role in the near future.

Integration into other major agendas: As explained injury and violence prevention has grown markedly, but it sometimes has worked too much in isolation from other fields and therefore remains absent from a number of high-level political debates. More attention could be gained for injury and violence prevention if the connection to other important public health and development issues was made. Highlighting the need for road safety to allow for more physical activity or less pollution, for violence prevention to contribute to reductions in HIV transmission or for child injury prevention to improve child survival, could generate additional attention and resources. Including injury prevention in other important political agendas such as poverty reduction and global warming is also needed. Efforts should be made to convince policy makers that investments in injury and violence prevention could contribute to achievements in other important agendas. This in turn would bring additional resources to strengthen violence and injury prevention work.

Building capacity: Capacity to address injuries and violence has increased dramatically in the past decades thanks to the efforts of many individuals and institutions, a growth in academic instruction and journal coverage, and programmes with a global reach covering technical exchange and cooperation. Examples of the latter include the Fogarty International Fellowships, TEACH-VIP and MENTOR-VIP. However, in many countries there is still a dearth of experts knowledgeable about the public health approach to violence and injury prevention. It is hoped that in coming years every school of public health will have formal training in injury prevention, that regional conferences will emerge to overcome the economic and language barriers associated with the series of World Conferences, that national training programmes develop, that MENTOR-VIP will have grown to allow for a larger number of people to be mentored and possibly expand to provide for institutional partnering.

Partnerships: Finally, a lot could be gained by continuing to foster solid partnerships. A number of such partnerships -such as the UN Road Safety Collaboration, the Violence Prevention Alliance, the Trauma and Emergency Care Services Network, the Road Traffic Injury Research Network, etc- have emerged in the past few years. The collaboration among the members of these networks has allowed for the pooling of knowledge and resources which have resulted in concrete products, but above all they have resulted in pooling political influence to create action that none of the partners could have achieved alone.

By supporting these additional activities while maintaining its current work, WHO will continue to support the growth of a vigorous and effective violence and injury prevention field that hopefully will benefit people everywhere.

Etienne G Krug*

\footnotetext{
* Director, Department of Injuries and Violence Prevention, World Health Organization, Geneva, Switzerland.
} 